\title{
Measurement and calculation of the two-dimensional backscattering Mueller matrix of a turbid medium
}

\author{
Brent D. Cameron \\ Biomedical Engineering Program, Texas A\&M University, College Station, Texas 77843-3120
}

M. J. Raković

Center for Theoretical Physics, Department of Physics, Texas A\&M University, College Station, Texas 77843-4242, and Institute of Physics, University of Belgrade, P.O. Box 57, 11001 Belgrade, Yugoslavia

Mehrübe Mehrübeoğlu

Department of Electrical Engineering, Texas A\&M University, College Station, Texas 77843-3128

George W. Kattawar

Center for Theoretical Physics, Department of Physics, Texas A\&M University, College Station, Texas 77843-4242

Sohi Rastegar, Lihong V. Wang, and Gerard L. Coté

Biomedical Engineering Program, Texas A\&M University, College Station, Texas 77843-3120

Received November 10, 1997

\begin{abstract}
We present both experimental and Monte Carlo-based simulation results for the diffusely backscattered intensity patterns that arise from illumination of a turbid medium with a polarized laser beam. A numerical method that allows the calculation of all 16 elements of the two-dimensional Muller matrix is used; moreover, it is shown that only seven matrix elements are independent. To validate our method, we compared our simulations with experimental measurements, using a turbid medium consisting of 2.02- $\mu$ m-diameter polystyrene spheres suspended in deionized water. By varying the incident polarization and the analyzer optics for the experimental measurements, we obtained the diffuse backscattering Mueller matrix elements. The experimental and the numerical results are in good agreement. (C) 1998 Optical Society of America

OCIS codes: $290.1350,290.7050,260.5430$.
\end{abstract}

A few recent studies demonstrated that one can measure information on the properties of a turbid medium by shining a polarized laser beam upon a sample and then analyzing the state of polarization of the diffusely backscattered light. The investigated applications of this technique include measurements for the average particle size, the scattering coefficient, and the anisotropy factor of particle suspensions ${ }^{1}$ as well as cloud diagnostics, ${ }^{2,3}$ the study of biological material, ${ }^{4-6}$ and the measurement of average photon path lengths. ${ }^{7}$

To achieve full experimental characterization of the optical properties of the sample under investigation, Hielscher et $a l .{ }^{6}$ used a Stokes vector-Mueller matrix approach to polarized light scattering. They generalized the concept of the effective Mueller matrix ${ }^{8}$ and measured the two-dimensional Mueller matrix of the backscattered light from a turbid medium. In a recent theoretical paper Ambirajan and Look ${ }^{9}$ used a Monte Carlo technique to study the multiple scattering of a polarized light beam from a plane-parallel medium. They investigated the degree of polarization of the diffuse light when the incident beam was right circularly polarized.

In this Letter our theoretical analysis is based on the assumption that the scattering of light is incoherent. The incoming narrow laser beam propagates downward along the $z$ axis and scatters from the medium located in the lower half-space. Let $\mathbf{P}_{\mathbf{0}}$ be the Stokes vector that corresponds to the power of the incident laser beam with respect to the $x-z$ reference plane and let $\mathbf{I}^{\mathbf{b s}}(\rho, \phi)$ be the Stokes vector that describes the radiance at the detector [i.e., at the point $(\rho, \phi)$ on the surface of the scattering medium]. Then, $\mathbf{I}^{\mathbf{b s}}(\rho, \phi)=$ $\mu_{s}{ }^{2} \mathbf{S}\left(\rho, \phi ; \mu_{s}, \mu_{T}\right) \mathbf{P}_{0}$, where $\mu_{s}$ and $\mu_{T}$ are the scattering and the extinction coefficients, respectively, and $\mathbf{S}$ can be seen as the effective backscattering Mueller matrix. If the scattering medium is homogeneous, the effective Mueller matrix takes the form

$$
\begin{gathered}
\mathbf{S}\left(\rho, \phi ; \mu_{s}, \mu_{T}\right)=\mathbf{R}(-\phi) \mathbf{L}\left(\rho_{s}, \bar{\omega}\right) \mathbf{R}(-\phi), \\
\mathbf{L}(\rho, \bar{\omega})=\sum_{n=2}^{\infty} \bar{\omega}^{n-2} \mathbf{L}_{n}\left(\rho_{s}\right), \quad \rho_{s}=\mu_{T} \rho, \quad \bar{\omega}=\frac{\mu_{s}}{\mu_{T}},
\end{gathered}
$$

where $\bar{\omega}$ is the single-scattering albedo and $\rho_{s}$ is the scaled radial distance. $\quad \mathbf{R}$ is the standard $4 \times 4$ matrix that rotates the reference plane. ${ }^{10}$ The term $\mathbf{L}_{n}$ in the above sum corresponds to backscattered light that has been scattered $n$ times. For example, the contribution of the double-scattered light consists of two terms $\mathbf{L}_{2}=\mathbf{L}_{2}{ }^{\prime}+\mathbf{L}_{2}{ }^{\prime \prime}$, where $\mathbf{L}_{2}^{\prime}$ corresponds to the light that is scattered twice before reaching the detector and $\mathbf{L}_{2}$ " corresponds to the light that is additionally reflected from the surface of the medium before the second scattering:

(C) 1998 Optical Society of America 


$$
\begin{aligned}
\mathbf{L}_{2}^{\prime}= & \int_{0}^{\infty} \int_{0}^{\infty} \frac{\mathrm{d} z_{1} \mathrm{~d} z_{2}}{r^{2}} \exp \left[-\left(z_{1}+z_{2}+r\right)\right] \\
& \times \mathbf{M}(\pi-\theta) \mathbf{M}(\theta), \\
r= & {\left[\rho_{s}{ }^{2}+\left(z_{1}-z_{2}\right)^{2}\right]^{1 / 2}, \quad \tan \theta=\frac{\rho_{s}}{z_{2}-z_{1}}, } \\
\mathbf{L}_{2}{ }^{\prime \prime}= & \int_{0}^{\infty} \int_{0}^{\infty} \frac{\mathrm{d} z_{1} \mathrm{~d} z_{2}}{r^{2}} \exp \left[-\left(z_{1}+z_{2}+r\right)\right] \\
& \times \mathbf{M}(\pi-\theta) T(\theta) \mathbf{M}(\pi-\theta), \\
r= & {\left[\rho_{s}{ }^{2}+\left(z_{1}+z_{2}\right)^{2}\right]^{1 / 2}, \quad \tan \theta=\frac{\rho_{s}}{z_{2}+z_{1}} . }
\end{aligned}
$$

In Eqs. (2), $\mathbf{M}(\theta)$ is the single-scattering Mueller matrix and $T(\theta)$ is the Fresnel matrix that describes the reflection from the surface of the medium. One can prove that each matrix $\mathbf{L}_{n}$, and therefore their sum $\mathbf{L}$, verifies the following important equality:

$$
\begin{aligned}
\mathbf{L}_{n}{ }^{t} & =\mathbf{P L}_{n} \mathbf{P}, \quad \mathbf{L}^{t}=\mathbf{P L P}, \\
\mathbf{P} & =\left[\begin{array}{cccc}
1 & 0 & 0 & 0 \\
0 & 1 & 0 & 0 \\
0 & 0 & -1 & 0 \\
0 & 0 & 0 & 1
\end{array}\right]
\end{aligned}
$$

Equation (3) holds provided that the single-scattering Mueller matrix $\mathbf{M}$ also satisfies the relation $\mathbf{M}^{t}=$ PMP. In our case, $\mathbf{M}$ is the familiar Mie scattering matrix. From the explicit form of the matrices $\mathbf{T}$ and $\mathbf{M}$, it follows that they also satisfy the above the relation (i.e., $\mathbf{M}^{t}=\mathbf{P M P}$ and $\mathbf{T}^{t}=\mathbf{P T P}$ ). ${ }^{11,12}$ From this relation and Eqs. (2), it follows that Eq. (3) holds for $\mathbf{L}_{2}$. For matrices $\mathbf{L}_{n}, n>2$, the forms of the corresponding integrals are similar to those in Eqs. (2), except that there are more integration variables and matrices in the product contained within the integral. The main difference is that the product within the integral contains, besides $\mathbf{T}$ and $\mathbf{M}$, the finite number of rotational matrices $\mathbf{R}$. However, the relation $\mathbf{R}^{t}=\mathbf{P R P}$ holds, and therefore one can prove that the matrices $\mathbf{L}_{n}$, for $n>2$, also satisfy Eq. (3).

Equation (3), together with Eq. (1), shows that not all elements of the matrix $\mathbf{S}$ are independent. In fact, there are only seven independent elements, $S_{11}, S_{12}$, $S_{14}, S_{22}, S_{23}, S_{24}$, and $S_{44}$; and the other nine,

$$
\begin{aligned}
& S_{13}=S_{12}\left(\varphi+\frac{\pi}{4}\right), \quad S_{21}=S_{12} \\
& S_{31}=S_{12}\left(\varphi-\frac{\pi}{4}\right), \quad S_{32}=S_{23}\left(\varphi \pm \frac{\pi}{4}\right) \\
& S_{33}=-S_{22}\left(\varphi \pm \frac{\pi}{4}\right), \quad S_{34}=S_{24}\left(\varphi-\frac{\pi}{4}\right) \\
& S_{41}=S_{14}, \quad S_{42}=S_{24}, \quad S_{43}=S_{24}\left(\varphi+\frac{\pi}{4}\right)
\end{aligned}
$$

can be obtained by simple rotations.
It should be mentioned that these important relations hold under much weaker conditions than those implied by our model (i.e., an optically inactive medium with spherical scatterers). It is sufficient that the scattering medium be invariant under rotations around the axis of the incident laser beam. Equations (4) also hold for an optically active medium, and the scattering particles can also be dichroic or birefringent, provided that they are randomly distributed.

To calculate numerically the effective Mueller matrix $\mathbf{S}$, we used the Monte Carlo method. Essentially, we discretized the integrals in the sum of Eqs. (1) and expressed $\mathbf{L}$ as a sum over a large number of randomly chosen photon trajectories, each defined by the collection of points at which the corresponding scattering event took place. The number of these points [which corresponds to the index in the sum of Eqs. (1)] was arbitrary. The trajectories were discarded if their contributions were below a suitable cutoff value. Each trajectory then gave its contribution to the total effective matrix $\mathbf{S}$. In this way we could calculate all matrix elements $S_{i j}$ simultaneously, unlike the experimental measurements, in which we had to combine results from several initial states of the incoming laser beam to determine the matrix elements.

A schematic view of the experimental apparatus used for collection of the diffuse backscattered images in shown in Fig. 1. The phantom that was utilized in this study was composed of a suspension of $2.02-\mu \mathrm{m}$ polystyrene spheres (Ernest F. Fullam, Latham, N.Y.). The sample was created by dilution of $0.075 \mathrm{ml}$ of a 10 -wt. \% suspension of polystyrene spheres with $15 \mathrm{ml}$ of deionized water. The index of refraction of the spheres was 1.59 , and the sample had a relative index of refraction $n / n_{w}$ of 1.192 , where $n_{w}$ is the index of refraction for water. The sample had a scattering coefficient $\left(\mu_{s}\right)$ of $11.88 \mathrm{~cm}^{-1}$ and an anisotropy factor $(\mathrm{g})$ of 0.912 at $632.8 \mathrm{~nm}$ and had a negligible absorption coefficient $\left(\mu_{a} \approx 0, \bar{\omega} \approx 1\right)$.

To determine each of the 16 experimental Mueller matrix elements, we took 49 images at various combinations of input and output analyzer polarization states. The details of the input and output

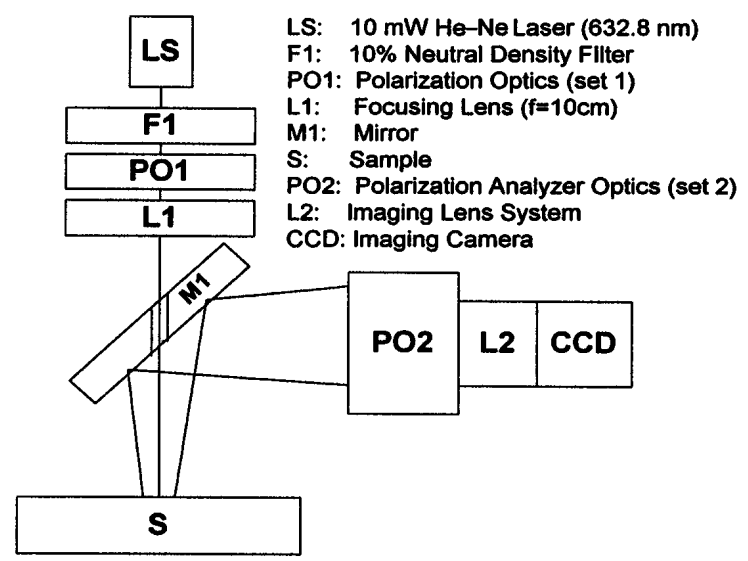

Fig. 1. Schematic diagram of experimental setup used for the Mueller matrix imaging of the diffusely backscattered light from the sample. 


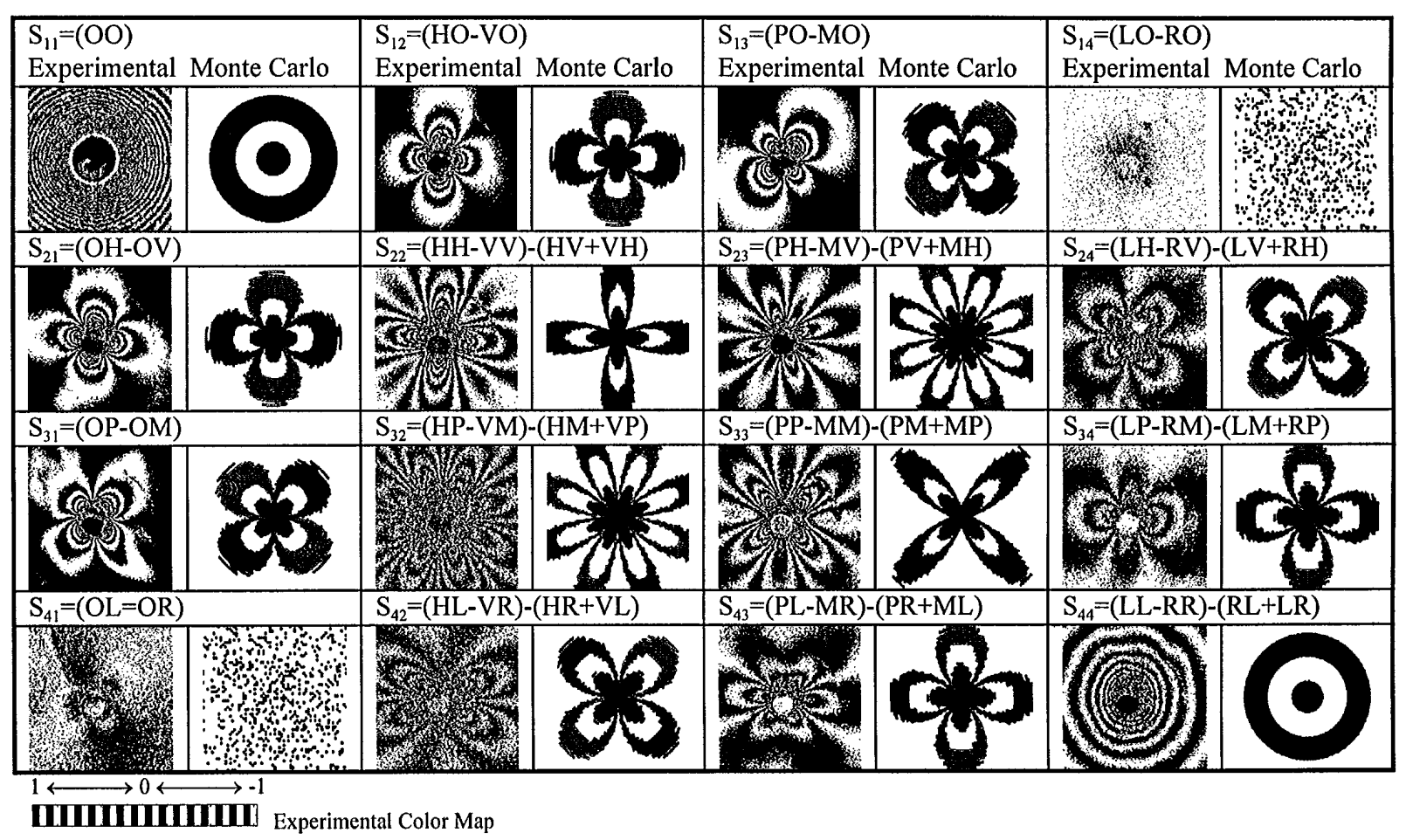

Fig. 2. Experimental and Monte Carlo-simulated diffusely backscattered Mueller matrix for a 2.02- $\mu \mathrm{m}$ polystyrene sphere suspension. The approximate size of each image is $1.6 \mathrm{~cm} \times 1.6 \mathrm{~cm}$. Each of the 16 experimental elements is calculated by addition or subtraction of a series of images. The individual images are represented by a two-letter combination that denotes the input polarization and the output analyzer orientation (i.e., HV denotes horizontal input polarized light and a vertical polarization analyzer). The corresponding symbols denoting polarization are $\mathrm{V}$, vertical; $\mathrm{H}$, horizontal; $\mathrm{P},+45^{\circ} ; \mathrm{M},-45^{\circ}$; R, right; L, left; and O, open or no polarization optics. For the Monte Carlo simulations, the average number of collisions per trajectory is 10 .

polarization states are indicated above each Mueller matrix element in Fig. 2 for computation of each of the experimental elements.

Note that for the images presented in Fig. 2 we chose the given color map to enhance the azimuthdependent patterns for comparison with the simulation results, and Fig. 2 does not illustrate the radial decay in the intensity especially well. The transport mean free path for the suspension used in our study, $m f p^{\prime}=1 /\left[\mu_{a}+\mu_{s}(1-g)\right]{ }^{4}$ was $0.967 \mathrm{~cm}$. It appears that, for distances approaching approximately two transport mean free paths from the input incident laser point, the azimuthal dependence of the patterns becomes less pronounced because multiple scatterings tended to randomize the polarization state of the light. Also, the radial falloff in the diffuse light intensity after approximately two transport mean free paths from the input incident laser point follows an exponential decay rate similar to that seen in the $S_{00}$ case, in which no polarizers were used. Other agreements seen in Fig. 2 for the experimental and the simulated results are the rotational relations [see Eqs. (4)] among the Mueller matrix elements.

Although the laser beam in the experiments was coherent, the light treated in the Monte Carlo simulations was incoherent. Because the correlation time of the laser-induced speckles is of the order of $10 \mathrm{~ms}$, the experimental images acquired with an exposure time of
$1.7 \mathrm{~s}$ have the speckle effect averaged out. Therefore, the coherence effect is not important in our images.

This research was partially supported by the Office of the Vice President for Research, Texas A\&M University; U.S. Office of Naval Research contract N0001495-1-0275; and the Whitaker Foundation.

\section{References}

1. A. H. Hielscher, J. R. Mourant, and I. J. Bigio, Appl. Opt. 36, 125 (1997).

2. A. I. Carswell and S. R. Pal, Appl. Opt. 19, 4123 (1980).

3. S. R. Pal and A. I. Carswell, Appl. Opt. 24, 3464 (1985).

4. S. L. Jacques, M. Ostemeyer, L. Wang, and D. Stephens, Proc. SPIE 2671, 199 (1996).

5. S. G. Demos and R. R. Alfano, Appl. Opt. 36, 150 (1997).

6. A. H. Hielscher, A. A. Eick, J. R. Mourant, and I. J. Bigio, Proc. SPIE 2976, 298 (1997).

7. M. Dogariu and T. Asakura, Opt. Eng. 35, 2234 (1996).

8. W. S. Bickel and W. M. Bailey, Am. J. Phys. 53, 468 (1985).

9. A. Ambirajan and D. C. Look, J. Quant. Spectrosc. Radiat. Transfer 58, 171 (1997).

10. R. Azzam and N. M. Bashara, Ellipsometry and Polarized Light (North-Holland, Amsterdam, 1977), p. 150.

11. H. C. van de Hulst, Light Scattering by Small Particles (Dover, New York, 1957).

12. G. W. Kattawar and C. N. Adams, Limnol. Oceanogr. 48, 1453 (1989). 